\title{
Trends und Megatrends in Business Intelligence
}

\section{Controlling Forum an der Hochschule Pforzheim}

Mobile BI, Cloud Computing, „Self-Service“ BI, Datenschutz - das sind die aktuellen Themen im Bereich der Controlling-Informationssysteme, also der Business Intelligence (BI), die beim letzten Controlling Forum der Hochschule Pforzheim Ende September auf der Tagesordnung standen. Dabei mussten den rund 20 Controllern, Managern und Wissenschaftlern die Aktualität und Relevanz des Themas kaum groß erklärt werden. BI als solches ist schon seit Jahren ein Standardthema unter Controllern. Aber aus den Entwicklungen in Sachen Internet und Mobile Devices ergeben sich derzeit eine Reihe von Konsequenzen für die Systeme. Und auch die aktuelle politische Diskussion zum Thema Ausspähung spielt eine Rolle, betrifft sie doch die Datensicherheit.

$\mathrm{Zu}$ Beginn gab Tobias Riedner, Analyst beim Business Application Research Center - Barc GmbH in Würzburg, einen Überblick über die BI-Trends. Er unterschied dabei zwischen solchen, die noch am Anfang des breiten Interesses stehen („earlymover Akzeptanz“), solchen mit schon breiter Marktrelevanz und einer Gruppe von Themen, die nicht neu, aber nach wie vor aktuell sind. Zur ersten Gruppe gehört beispielsweise „collaborative BI“, d. h. die Integration von BI mit anderen Tools wie Web 2.0-Technologien. „Big Data“, Self-Service BI - also der Betrieb von BI und insbesondere die Erstellung der Reports durch die Fachabteilung ohne Hilfe des IT-Bereichs - und Mobile BI gehören zur zweiten Gruppe. Planung und Forecasting sind Beispiele der dritten Kategorie.

Anschließend stellten Bernhard Webler von Microstrategy Deutschland und Jörg Rose von Cubeserv aktuelle Entwicklungen aus Mobile BI vor. Die mit Live-Demos untermauerten Möglichkeiten der Nutzung mobiler Endgeräte, vor allem der Zugriff auf die Daten von jedem beliebigen Ort und die Möglichkeiten der Analyse, beeindruckten durchaus. Kritisch hinterfragten die Teilnehmer allerdings die eventuell langen Antwortzeiten im praktischen Einsatz und die Lesbarkeit groBer Reports auf kleinen Tablet- und Smartphone-Bildschirmen. Während die Referenten die Bedenken hinsichtlich Tablets ausräumen konnten, galt das nur bedingt für die Smartphones. Für deren noch kleineren Screen sind die meisten Reports nicht optimiert. Vor allem aber wurde die durch
Mobile BI entstehende Doppelarbeit kontrovers diskutiert. Reports müssen einmal für die konventionellen Anwendungen erstellt werden und zusätzlich für die mobilen Applikationen. Diesem Zusatzaufwand steht aus Sicht vieler Teilnehmer nur ein begrenzter Zusatznutzen gegenüber. Schon heute können sie via Intranet / Internet auf herkömmlichen BISystemen von vielen Orten arbeiten - ein Notebook und ein mittlerweile fast überall verfügbares WLAN-Netz reichen dazu aus. Viele kamen daher zu dem Schluss, dass mobile Applikationen beispielsweise für den Vertriebsaußendienst sinnvoll sein mögen, für das Controlling (noch) nicht.

Auch die Vorteile von Cloud Computing sind für das Controlling differenziert zu sehen. Wie Markus Sümmchen, Geschäftsführer des Softwareunternehmens white duck $\mathrm{GmbH}$, ausführte, realisieren sich insbesondere die von Cloud Computing erhofften Kostenvorteile oft nicht. Cloud Computing erspart nur bei variabler, stark schwankender Nutzungsintensität Kosten, da das Unternehmen dann nicht stets die gesamten Kapazitäten vorhalten muss. Entwarnung gab er aber zumindest teilweise beim Thema Datensicherheit. Zwar ist der Schutz der Daten beim Transfer zwischen Unternehmen und Cloud naturgemäß problematisch. Andererseits stuft er das Niveau an Datensicherheit, das ein professioneller Cloud-Anbieter Unternehmen bieten kann, deutlich höher ein als das einer internen IT.

Am Ende der Veranstaltung zogen die Teilnehmer ein einhelliges Fazit: Die Aussichten im BI-Bereich sind für Controller teilweise faszinierend. Es ist aber nicht alles Gold, was auf den ersten Blick zu glänzen scheint.

Damit ging auch bei diesem mehrmals im Jahr stattfindenden Controlling Forum das Konzept dieser Veranstaltungsreihe voll auf: Seminare mit Workshop-Charakter anzubieten, die es erlauben, auch auf individuelle Fragen der Teilnehmer einzugehen.

Mehr Informationen zu dieser Veranstaltungsreihe finden Sie unter www.controlling.biz.

Prof. Dr. Joachim Paul, Pforzheim 